\title{
Embedded System for Automatic Condensation Control of the Car
}

\author{
Dmitriy Lee1, Yong-Wook Bae1, Neung-Ho Lee², and Hee-Don Seo',++
}

\begin{abstract}
In this study, we designed an embedded system for automatic condensation control(ESCC) of the car. This system heats the car glasses as and when it is needed that makes driving safer and convenient. The system was built on an ATmega128L central processing unit(CPU), using high-performance electrically erasable programmable read-only memory(EEPROM) complex programmable logic device(CPLD) ATF1504AS, using which an ESCC algorithm has been proposed. The source code was written in C language. The algorithm of work was written using the dew-point table. This system not only clears the condensation on the glass but also averts condensation. The designed ESCC system begins working once the input information comes close to the dew-point table information. This device enables a wider field of view, thereby increasing safety.
\end{abstract}

Keywords : Embedded System for Automatic Condensation Control(ESCC)

\section{INTRODUCTION}

Air contains water vapor in various percentages. The dew-point indicates the amount of moisture in the air. The higher the dew-point, the higher the moisture content of the air at a given temperature. In other words, the dew-point of humid air is higher than the dew-point of dry air.

The dew-point temperature is defined as the temperature up to which air would have to cool(at constant pressure and constant water vapor content) in order to reach saturation. Saturation state is reached when air holds the maximum amount of water vapor possible at the current temperature and pressure.

The condensation of water vapor begins when the temperature of air is lowered to its dew-point and beyond. The dew-point, similar to the other measures of humidity, can be calculated from readings taken by a hygrometer. Condensation is a very common phenomenon in everyday life. Although it does not leave durable signs on windowpanes, it can be very unpleasant because it reduces visibility, especially during driving. When water vapor in air comes into contact with cold surfaces, the vapor

${ }^{1}$ Department of Electronic Engineering, Yeungnam University

${ }^{2}$ Department of Electronic Engineering, Korea Polytechnic College

+Corresponding author: hdseo@ynu.ac.kr

(Recevied: Oct. 27, 2011, Revised : Jan. 3, 2012, Accepted : Jan. 11, 2012) condenses on the cooler surface of the glass creating a foggy effect. This condensation is annoying during driving, and it indicates excessive humidity inside the car.

In recent times, modern cars have a powerful electronic heating system for the passenger compartment and some form of heater attached to the rear glass.

Many new cars and trucks now have automatic temperature control(ATC) systems that not only regulate cooling but also regulate heating for year-round passenger comfort. Most of these systems have their own built-in computers that are integrated into the control panel head, located elsewhere, or integrated into the body control module.

Most ATC systems have a manual override mode or semiautomatic mode that allows the driver to control most of its functions. However, some manual control modes must pass through the computer and must be acceptable to the operating logic of the ATC system.

Automobile manufacturers develop their own operating logic that varies from one model to another and from one year to the next. The logic dictates whether the system must go into the recirculation mode to maximize cooling when a vehicle is first started on a hot day or whether it must not. Most systems do not turn on the airconditioning $(\mathrm{A} / \mathrm{C})$ compressor if ambient temperatures are below freezing point, although they may run the $\mathrm{A} / \mathrm{C}$ 
compressor when the system is in the defrost mode to dehumidify air[1].

In this article, we design an embedded system for automatic condensation control(ESCC) of the car.

We propose an algorithm for the functioning of the ESCC, which can avert condensation of car glasses. We apply our proposed algorithm to real-time situations to verify its effectiveness by comparing with calculation results. The system developed in this study is a new approach, and so far analog embedded systems have not been designed or developed for condensation control in vehicles.

The proposed ESCC system heats the car glasses when needed and makes driving safer and more convenient. It is built on an ATmega128L central processing unit(CPU), using high-performance electrically erasable programmable read-only memory(EEPROM) complex programmable logic device(CPLD) ATF1504AS.

The ESCC system not only clears the condensation from the glass but also averts condensation. The system begins working when the input information comes close to the dew-point table information. After several experiments, we found that this system can increase driver safety level significantly by preventing condensation on the car glasses.

\section{ESCC DESIGN AND THE PROPOSED ALGORITHM}

The ESCC system is a new device that helps improve visibility, thereby increasing driver safety. This system is designed such that it controls the breathing of the car's glasses and detects the temperature and humidity of the passenger compartment. The proposed design is discussed in the following section.

\subsection{ESCC design}

The ESCC receives data regarding the glass temperature and the inside temperature and humidity of the passenger compartment. The input information to the system is based on the data and the out-of-ratio temperature and humidity. The ESCC includes the following functions: clock, alarm, three modes of operation(automatic, timer, and manual), SMART-electrical energy control system, temperature management(glasses), temperature(inside, glasses) and humidity information, and adaptive learning functions. The clock displays a numeric representation of time using the 24-h clock system.(The 24-h clock is a convention used for timekeeping in which the day runs from midnight to midnight and is divided into $24 \mathrm{~h}$, numbered from 0 to 23 . The alarm of the ESCC is designed to switch on the glass heating system at a specific pre-defined time. The selfteaching mode makes this system almost universal, that is, it works in any weather conditions appropriately[2]. This mode stores information for the future and informs the ESCC regarding the current temperature and humidity inside the car.

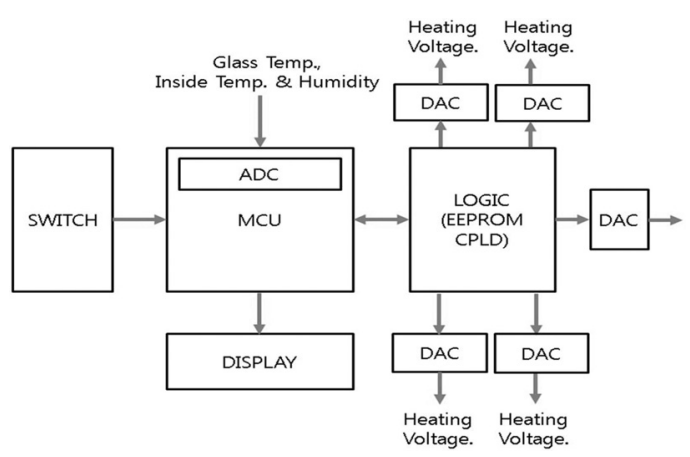

Fig.1. Block diagram of ESCC.

Figure 1 represents a block diagram of the ESCC. The primary function of the microcontroller unit(MCU) is to measure the temperatures of the each of the car's glasses and the inside temperature and humidity of the car. The MCU also makes a decision whether to heat or not to heat each window glass based on the dew-point temperature and the input data. The MCU includes an 8-channel, 10-bit analog-to-digital convertor(ADC) with an optional differential-input stage with programmable gain and six software selectable power saving modes. A CPLD chip extends the $\mathrm{I} / \mathrm{O}$ of the MCU and the output of the five digital-to-analog convertor(DAC). The five DAC generates the voltage for heating the window glasses.

\subsection{Algorithm}

The electronic control unit(ECU) of the car is designed to make driving convenient and safe. However, the ECU does not provide a solution to the problem of condensation before starting the engine. The ESCC takes care of clearing 
car glasses at all times of the day and in all types of weather conditions. The algorithm uses an exclusive and smart energy-optimization function that ensures that ESCC works all $24 \mathrm{~h}$ in a day. The source code was written in $\mathrm{C}$ language. The algorithm was written using a dew-point table.

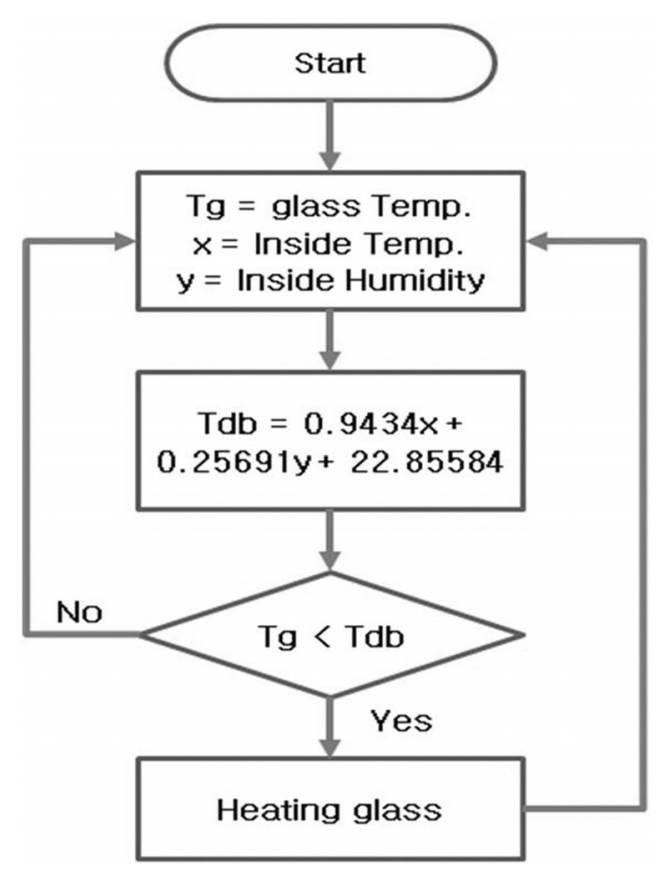

Fig. 2. Flowchart of ESCC.

The ESCC performs the algorithm represented by Figure 2 with temperature of heating battery, and baking time.

The system begins to heat the window, only when the critical moment is reached. The critical moment is defined as the moment at which the coefficients of temperature and humidity are close to the dew-point data. The heating proceeds step by step and does not allow accumulation of moisture on the glass. The ESCC has many benefits, such as easy integration with one of the already installed ECUs and support of the alarm function(which helps save energy)[3].

Figure 3 is a graphical representation of the glass heating process, with a dew-point equal to $-6{ }^{\circ} \mathrm{C}$ (temperature $=10$ ${ }^{\circ} \mathrm{C}$, humidity $=30 \%$ ).

The system turns on the heater $3{ }^{\circ} \mathrm{C}$ before the dew-point is reached, and heats up every $0.3{ }^{\circ} \mathrm{C}$ until $12 \mathrm{~V}$, thereby averting condensation. The ESCC supports a "SLEEP" mode during nighttime when the car is not being used for 5 h - $6 \mathrm{~h}$. This function helps save energy. The alarm function returns the system to the online mode. Even before the driver comes back to the vehicle, all the car glasses will be clear.

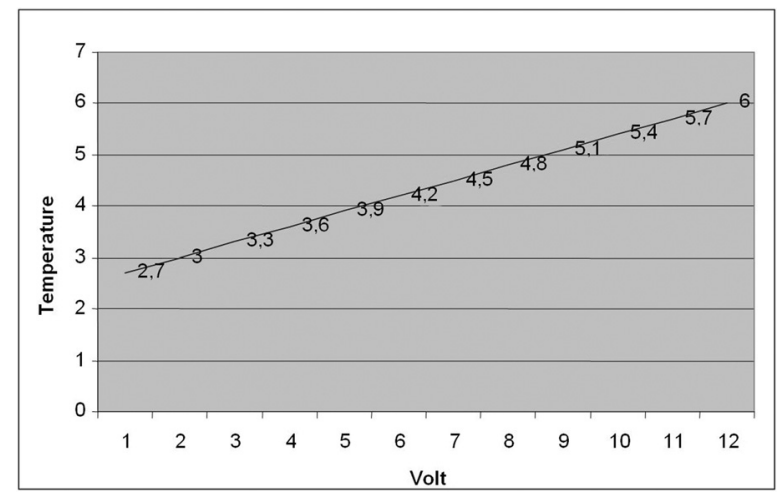

Fig. 3. Graph of glass heating.

\section{EXPERIMENTS AND RESULTS}

In this section, the ESCC is verified using experiments.

An EPU(electric power unit) was designed to increase the voltage of the automobiles. The EPU increases and stabilizes voltage from $12 \mathrm{~V}$ to $5 \mathrm{~V}$, and it showed perfect results without any delays and problems during all the experiments. The designed ESCC works for $96 \mathrm{~h}$ in online mode without errors.

\subsection{Implementation of ESCC}

The ESCC was built on an ATmega128L CPU, EEPROM CPLD ATF1504AS, DAC0800, and other systems as shown in Figure 4.

The ATmega128L has onboard an 8-channel 10-bit ADC[4].

The ATmega128L provides the following features: 128 $\mathrm{KB}$ of in-system programmable flash with read-whilewrite capabilities, $4 \mathrm{~KB}$ EEPROM, $4 \mathrm{~KB}$ static randomaccess memory(SRAM), 53 general purpose I/O lines, 32 general purpose working registers, real-time counter(RTC), 2 universal asynchronous receiver/transmitters(USARTs), an 8-channel, 10-bit ADC with optional differential-input stage with programmable gain, and six software selectable power saving modes[5]. 


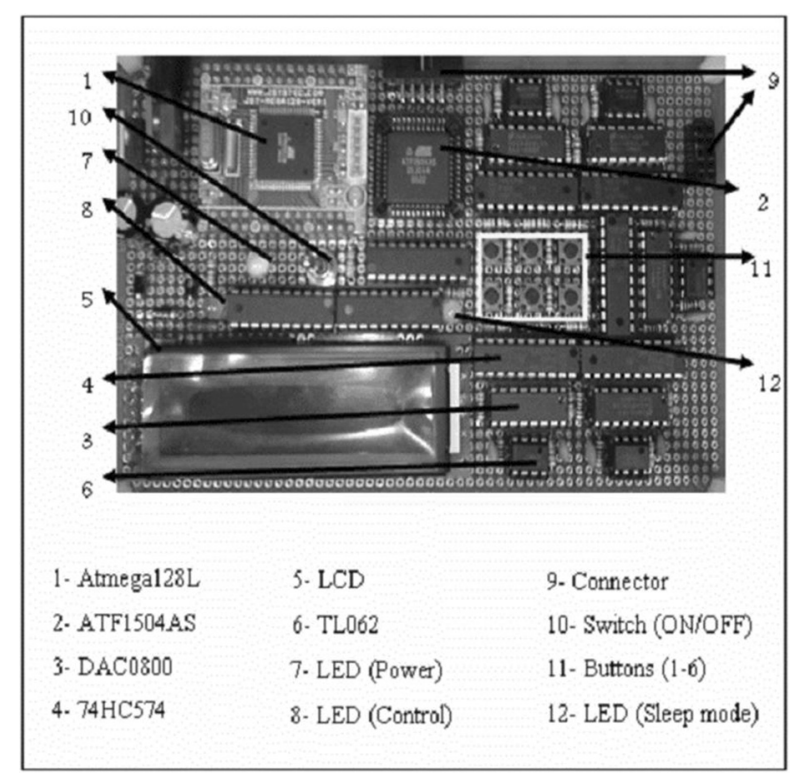

Fig. 4. ESCC of the car.

The idle mode stops the CPU while allowing the SRAM, timer/counters, serial peripheral interface(SPI) port, and interrupt system to continue functioning. The power-down mode saves the register contents but freezes the oscillator, disabling all other chip functions until the next interrupt or hardware reset. In the power-save mode, the asynchronous timer continues to run, allowing the user to maintain a timer base, while the rest of the device is sleeping. The ADC noise reduction mode stops the CPU and all the I/O modules except the asynchronous timer and the $\mathrm{ADC}$, to minimize switching noise during $\mathrm{ADC}$ conversions. In the standby mode, the crystal/resonator oscillator continues running, while the rest of the device sleeps. This allows very quick start-up and low power consumption. In the extended standby mode, both the main oscillator and the asynchronous timer continue to run[6].

The ATF1504AS is a high-performance, high-density CPLD that utilizes Atmel's proven electrically erasable technology. With 32 logic macrocells and up to 36 inputs, it easily integrates logic from several transistor-transistor logic(TTL), small-scale integration(SSI), medium-scale integration(MSI), large-scale integration(LSI), and classic programmable logic device(PLD) units.

The DAC0800 series are monolithic 8-bit high-speed current-output digital-to-analog converters featuring typical settling times of $100 \mathrm{~ns}$. When used as a multiplying DAC, monotonic performance over a 40 to 1 reference current range is possible.

The DAC0800 series also features high-compliance complementary current outputs to allow differential output voltages of $20 \mathrm{Vp}$-p with simple resistor loads. The system also allows to be tuned by 6 buttons and $16 \times 2$ liquid crystal display(LCD).

\subsection{Identification of the Dew-Point Temperature Equation}

The data presented in Table 1 was collected from the Internet, which represents experimental results in real-time conditions[7].

The plane equation is assumed as follows:

$$
a x+b y+c=T_{d p}
$$

Applying least squares method using Table 1, the following equation was obtained:

$$
0.9434 x+0.256915 y+22.85584=T_{d p}
$$

where

$$
\begin{aligned}
\mathrm{T}_{\mathrm{dp}} & =\text { dew-point temperature } \\
\mathrm{x} & =\text { temperature in the car } \\
\mathrm{y} & =\text { humidity }
\end{aligned}
$$

Table 2 shows the squared error of dew-point temperature when equation (2) is used. The maximum squared error is $\sqrt{5.581}$.

Table 1. Dew-point table from the internet

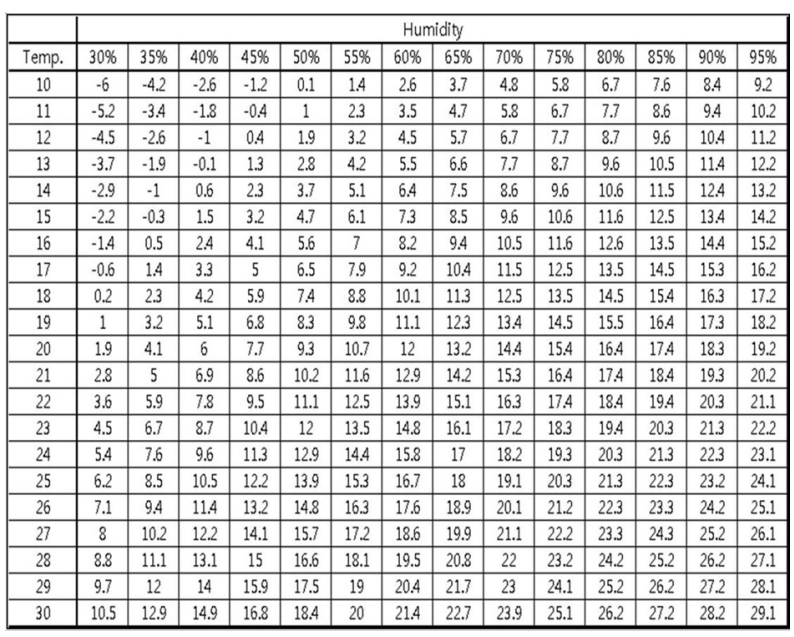


Table 2. Dew-point equation errors in squares

\begin{tabular}{|c|c|c|c|c|c|c|c|c|c|c|c|c|c|c|}
\hline & \multicolumn{14}{|c|}{ Humidity } \\
\hline emp. & $30 \%$ & $35 \%$ & $40 \%$ & $45 \%$ & $50 \%$ & $55 \%$ & $60 \%$ & \begin{tabular}{|l|}
$65 \%$ \\
\end{tabular} & $70 \%$ & $75 \%$ & $80 \%$ & $85 \%$ & $90 \%$ & $95 \%$ \\
\hline 10 & 0.036 & \begin{tabular}{|l|l}
0.107 \\
\end{tabular} & \begin{tabular}{|l|l|}
0.413 \\
\end{tabular} & \begin{tabular}{|l|l|}
0.574 \\
\end{tabular} & 0.598 & 0.622 & \begin{tabular}{|l|l|}
0.496 \\
\end{tabular} & 0.270 & 0.112 & \begin{tabular}{|l|}
0.003 \\
\end{tabular} & \begin{tabular}{|l|l|}
0.112 \\
\end{tabular} & \begin{tabular}{|l|l|}
0.517 \\
\end{tabular} & 1.448 & 2.849 \\
\hline 11 & 0.104 & 0.037 & 0.259 & 0.389 & \begin{tabular}{|l|l|}
0.547 \\
\end{tabular} & 0.570 & \begin{tabular}{|l|l|l|} 
\\
\end{tabular} & 0.343 & 0.161 & 0.000 & \begin{tabular}{|l|l|}
0.072 \\
\end{tabular} & \begin{tabular}{|l|l|} 
\\
\end{tabular} & \begin{tabular}{|l|l|}
1.293 \\
\end{tabular} & 2.630 \\
\hline 12 & . & \begin{tabular}{|l|l|l}
0.004 \\
\end{tabular} & \begin{tabular}{|l|l|}
0.141 \\
\end{tabular} & \begin{tabular}{|l|l|}
0.240 \\
\end{tabular} & \begin{tabular}{|l|}
0.498 \\
\end{tabular} & \begin{tabular}{|l}
0.520 \\
\end{tabular} & \begin{tabular}{|l|l|}
0.543 \\
\end{tabular} & 0.425 & \begin{tabular}{|l|l|l|}
0.104 \\
\end{tabular} & \begin{tabular}{|l|}
0.007 \\
\end{tabular} & \begin{tabular}{|l|l|} 
\\
\end{tabular} & \begin{tabular}{|l|l|}
0.344 \\
\end{tabular} & \begin{tabular}{|l|l|}
1.147 \\
\end{tabular} & \\
\hline 13 & 476 & 0.030 & \begin{tabular}{|l|l|}
0.116 \\
\end{tabular} & 0.209 & 0.452 & 0.620 & \begin{tabular}{|l|l|}
0.645 \\
\end{tabular} & 0.382 & 0.188 & 0.022 & 0.055 & \begin{tabular}{|l|l|}
0.384 \\
\end{tabular} & 1.009 & \begin{tabular}{|l}
2.217 \\
\end{tabular} \\
\hline 14 & 0.678 & 0.043 & \begin{tabular}{|l|l|}
0.012 \\
\end{tabular} & 0.274 & \begin{tabular}{|l|l|}
0.408 \\
\end{tabular} & 0.568 & 0.592 & 0.342 & 0.160 & 0.013 & 0.029 & $\begin{array}{l}0.306 \\
\end{array}$ & \begin{tabular}{|l|l|}
0.880 \\
\end{tabular} & 2.024 \\
\hline 15 & 117 & \begin{tabular}{|l|l|}
0.195 \\
\end{tabular} & 0.005 & \begin{tabular}{|l}
0.239 \\
\end{tabular} & \begin{tabular}{|l|}
0.497 \\
\end{tabular} & \begin{tabular}{|l|l|}
0.673 \\
\end{tabular} & \begin{tabular}{|c|}
0.541 \\
\end{tabular} & 0.424 & 0.218 & \begin{tabular}{|l|}
0.033 \\
\end{tabular} & 0.011 & \begin{tabular}{|l|}
0.237 \\
\end{tabular} & \begin{tabular}{|l|l|}
0.760 \\
\end{tabular} & 1.840 \\
\hline 16 & 1.418 & \begin{tabular}{|l|l|} 
\\
\end{tabular} & 0.002 & 0.208 & \begin{tabular}{|l|l}
0.450 \\
\end{tabular} & 0.619 & \begin{tabular}{|l|l|l} 
\\
\end{tabular} & \begin{tabular}{|l|l|}
0.381 \\
\end{tabular} & \begin{tabular}{|l}
0.187 \\
\end{tabular} & \begin{tabular}{|l|l|}
0.062 \\
\end{tabular} & 0.001 & 0.117 & 0.649 & 1.664 \\
\hline 17 & 1.754 & \begin{tabular}{|l|l}
0.371 \\
\end{tabular} & 0.000 & \begin{tabular}{|l}
0.178 \\
\end{tabular} & \begin{tabular}{|l|l|} 
\\
\end{tabular} & \begin{tabular}{|l}
0.567 \\
\end{tabular} & \begin{tabular}{|c|}
0.590 \\
\end{tabular} & \begin{tabular}{|l|}
0.467 \\
\end{tabular} & 0.249 & 0.046 & 0.005 & \begin{tabular}{|l|l|}
0.126 \\
\end{tabular} & \begin{tabular}{|l|l|}
0.704 \\
\end{tabular} & 1.498 \\
\hline 18 & 2.216 & 0.413 & \begin{tabular}{|l|l|}
0.001 \\
\end{tabular} & \begin{tabular}{|l|l|}
0.151 \\
\end{tabular} & \begin{tabular}{|l|l|}
0.364 \\
\end{tabular} & \begin{tabular}{|l|l}
0.517 \\
\end{tabular} & \begin{tabular}{|l|l|}
0.539 \\
\end{tabular} & 0.422 & 0.320 & \begin{tabular}{|l|l|}
0.079 \\
\end{tabular} & 0.000 & \begin{tabular}{|l|}
0.151 \\
\end{tabular} & \begin{tabular}{|l|l|}
0.594 \\
\end{tabular} & 1.340 \\
\hline 19 & 2.534 & \begin{tabular}{|l|l}
0.457 \\
\end{tabular} & \begin{tabular}{|l|}
0.004 \\
\end{tabular} & \begin{tabular}{|l|l|}
0.126 \\
\end{tabular} & \begin{tabular}{|l|l|} 
\\
\end{tabular} & \begin{tabular}{|l|l}
0.617 \\
\end{tabular} & \begin{tabular}{|l|}
0.641 \\
\end{tabular} & \begin{tabular}{|l|l|}
0.513 \\
\end{tabular} & \begin{tabular}{|l}
0.283 \\
\end{tabular} & \begin{tabular}{|c|}
0.120 \\
\end{tabular} & 0.004 & \begin{tabular}{|l|l|}
0.104 \\
\end{tabular} & \begin{tabular}{|l|l|l}
0.449 \\
\end{tabular} & 1.191 \\
\hline 20 & 2.642 & \begin{tabular}{|l|l}
0.504 \\
\end{tabular} & \begin{tabular}{|l|}
0.009 \\
\end{tabular} & \begin{tabular}{|l|l|}
0.103 \\
\end{tabular} & \begin{tabular}{|l|l|} 
\\
\end{tabular} & \begin{tabular}{|l}
0.565 \\
\end{tabular} & 0.588 & 0.466 & \begin{tabular}{|l|l}
0.588 \\
\end{tabular} & \begin{tabular}{|l|}
0.098 \\
\end{tabular} & \begin{tabular}{|l|}
0.001 \\
\end{tabular} & 0.065 & 0.410 & 1.050 \\
\hline 21 & 2.753 & 0.553 & 0.016 & 0.082 & 0.363 & \begin{tabular}{|l|l}
0.516 \\
\end{tabular} & \begin{tabular}{|l|l|}
0.538 \\
\end{tabular} & 0.561 & 0.318 & \begin{tabular}{|l|}
0.144 \\
\end{tabular} & 0.009 & \begin{tabular}{|l|l|}
0.036 \\
\end{tabular} & \begin{tabular}{|l|l|}
0.329 \\
\end{tabular} & 0.919 \\
\hline 22 & 3.214 & 0.604 & 0.026 & 0.064 & \begin{tabular}{|l|}
0.324 \\
\end{tabular} & 0.468 & 0.640 & 0.511 & 0.398 & \begin{tabular}{|l|}
0.199 \\
\end{tabular} & 0.026 & \begin{tabular}{|l|l|}
0.015 \\
\end{tabular} & \begin{tabular}{|l|l|}
0.258 \\
\end{tabular} & 0.98 \\
\hline 23 & 3.336 & \begin{tabular}{|l|l|}
0.830 \\
\end{tabular} & \begin{tabular}{|l|}
0.038 \\
\end{tabular} & 0.048 & \begin{tabular}{|l|}
0.286 \\
\end{tabular} & \begin{tabular}{|l|l}
0.563 \\
\end{tabular} & \begin{tabular}{|l|}
0.587 \\
\end{tabular} & 0.611 & 0.356 & \begin{tabular}{|c|}
0.170 \\
\end{tabular} & 0.052 & 0.025 & \begin{tabular}{|l|l|}
0.195 \\
\end{tabular} & 0.682 \\
\hline 24 & 3.460 & 0.893 & 0.053 & 0.035 & \begin{tabular}{|l|}
0.251 \\
\end{tabular} & \begin{tabular}{|l|l|l}
0.514 \\
\end{tabular} & \begin{tabular}{|l|l|}
0.693 \\
\end{tabular} & 0.559 & 0.440 & \begin{tabular}{|l|}
0.229 \\
\end{tabular} & \begin{tabular}{|l|l|}
0.038 \\
\end{tabular} & \begin{tabular}{|l|l|}
0.008 \\
\end{tabular} & \begin{tabular}{|l|l|}
0.141 \\
\end{tabular} & \begin{tabular}{|l}
0.739 \\
\end{tabular} \\
\hline 25 & 3.976 & \begin{tabular}{|l}
0.957 \\
\end{tabular} & \begin{tabular}{|l|}
0.069 \\
\end{tabular} & \begin{tabular}{|l|l|}
0.023 \\
\end{tabular} & \begin{tabular}{|l|l|} 
\\
\end{tabular} & \begin{tabular}{|l|l}
0.467 \\
\end{tabular} & \begin{tabular}{|l|l|}
0.638 \\
\end{tabular} & 0.663 & 0.396 & \begin{tabular}{|l|}
0.297 \\
\end{tabular} & \begin{tabular}{|c|}
0.068 \\
\end{tabular} & \begin{tabular}{|l|}
0.001 \\
\end{tabular} & \begin{tabular}{|l|}
0.167 \\
\end{tabular} & \begin{tabular}{|l}
0.629 \\
\end{tabular} \\
\hline 26 & 4.111 & \begin{tabular}{|l|l|}
1.024 \\
\end{tabular} & \begin{tabular}{|l|l|}
0.088 \\
\end{tabular} & \begin{tabular}{|l|l|l}
0.048 \\
\end{tabular} & 0.285 & \begin{tabular}{|l}
0.562 \\
\end{tabular} & \begin{tabular}{|l|l|}
0.585 \\
\end{tabular} & 0.603 & 0.484 & \begin{tabular}{|l|l|}
0.261 \\
\end{tabular} & \begin{tabular}{|l|l|}
0.107 \\
\end{tabular} & 0.002 & \begin{tabular}{|l|l|}
0.117 \\
\end{tabular} & \begin{tabular}{|l}
0.529 \\
\end{tabular} \\
\hline 27 & 4.249 & \begin{tabular}{|l|l|}
1.313 \\
\end{tabular} & \begin{tabular}{|l|l|}
0.185 \\
\end{tabular} & \begin{tabular}{|l|l}
0.034 \\
\end{tabular} & 0.250 & \begin{tabular}{|l|l}
0.512 \\
\end{tabular} & 0.691 & \begin{tabular}{|l|l}
0.717 \\
\end{tabular} & 0.581 & \begin{tabular}{|l|}
0.334 \\
\end{tabular} & \begin{tabular}{|l|l|} 
& 0.154
\end{tabular} & 0.012 & \begin{tabular}{|l|l|}
0.076 \\
\end{tabular} & \begin{tabular}{|l}
0.437 \\
\end{tabular} \\
\hline 28 & 4.818 & \begin{tabular}{|l|}
1.391 \\
\end{tabular} & \begin{tabular}{|l|}
0.215 \\
\end{tabular} & 0.023 & \begin{tabular}{|l|}
0.218 \\
\end{tabular} & 0.465 & 0.636 & 0.661 & 0.531 & 0.415 & 0.129 & \begin{tabular}{|c|c|} 
\\
\end{tabular} & \begin{tabular}{|l|l|}
0.044 \\
\end{tabular} & 0.3 \\
\hline 29 & 4.967 & 1.472 & 0.248 & 0.014 & 0.188 & 0.421 & 0.584 & 0.607 & 0.632 & \begin{tabular}{|l|}
0.372 \\
\end{tabular} & 0.181 & 0.020 & 0.021 & \begin{tabular}{|l|l|}
0.273 \\
\end{tabular} \\
\hline 30 & 5.581 & \begin{tabular}{|l}
1.555 \\
\end{tabular} & \begin{tabular}{|l|}
0.283 \\
\end{tabular} & \begin{tabular}{|l|l}
0.007 \\
\end{tabular} & \begin{tabular}{|l|}
0.159 \\
\end{tabular} & \begin{tabular}{|l|l|}
0.511 \\
\end{tabular} & 0.689 & \begin{tabular}{|l}
0.715 \\
\end{tabular} & 0.579 & \begin{tabular}{|l|}
0.458 \\
\end{tabular} & 0.242 & \begin{tabular}{|c|c|}
0.043 \\
\end{tabular} & 0.006 & 0.213 \\
\hline
\end{tabular}

Figure 5 represents the relationship or dependence between dew-point temperature and humidity.

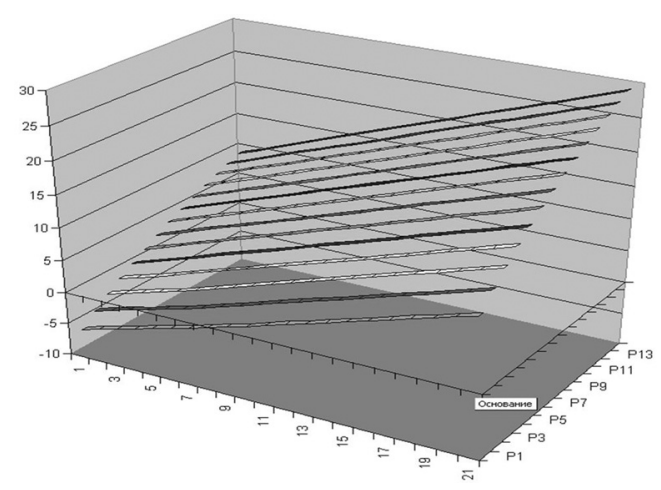

Fig. 5. Relationship between dew-point temperature and humidity.

\subsection{ESCC Analysis Results}

The data from Table 1 can be used to compare the working of the device against real-time situations.

Figure 6 shows the voltage difference between ideal and real-time working situations. This figure shows that the working of the ESCC in real time is almost the same as in the ideal case. Several experiments were conducted and most of the results were similar to the initial experimental results. The temperature of glass was increased manually almost to dew-point temperature.

This experiment shows the case of the system at the critical error point(Fig. 7). The maximum value of error is $\sqrt{5.581}$ (temperature inside the car compartment is $30{ }^{\circ} \mathrm{C}$, humidity is $30 \%$ ).

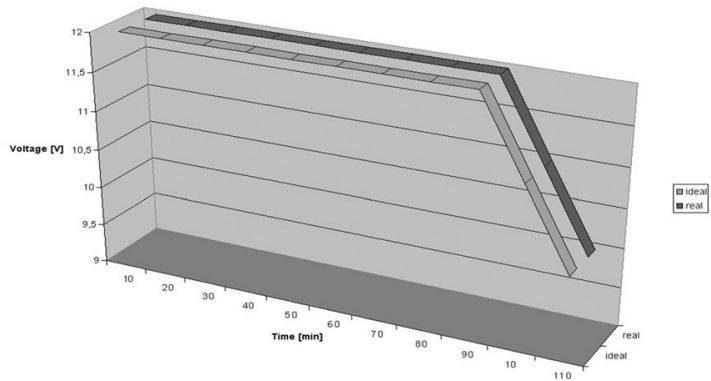

Fig. 6. Difference of voltage between ideal and real-time working situations.

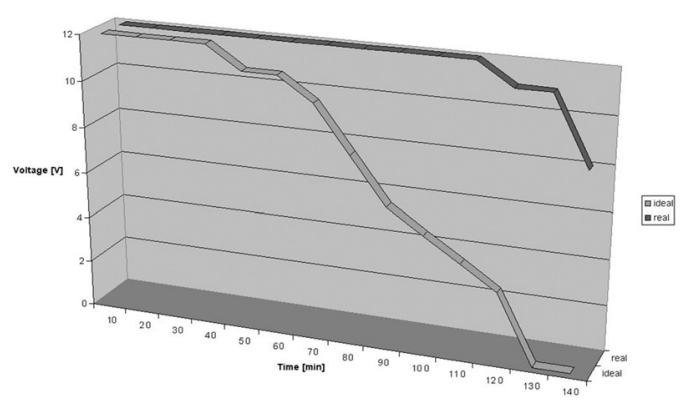

Fig. 7. Voltage difference between ideal and real-time working situations(at critical error point).

Figure 7 shows that the algorithm of simulation and algorithm in real situation is different. However, the ESCC averts the condensation even in this case. The system began to heat the car glasses a little earlier than it did in the ideal case. It means that the system began spending energy with full power, when it has to heat only by $11 \mathrm{~V}$. However, as shown in Figure 7, the glass heating began earlier too.

In Figure 8, the temperature inside the car compartment is $30{ }^{\circ} \mathrm{C}$, humidity is $30 \%$, and the temperature of glass is changeable: $8.9{ }^{\circ} \mathrm{C}$ to $14.2{ }^{\circ} \mathrm{C}$.

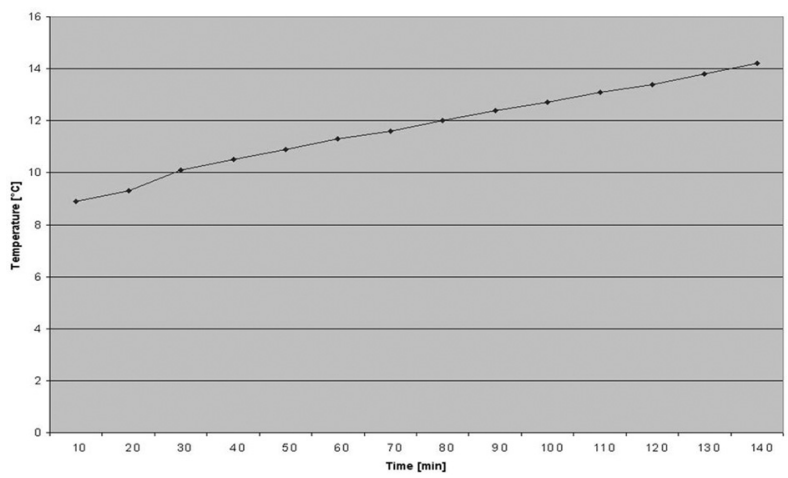

Fig. 8. Temperatures values (in critical error point). 
In conclusion, based on these experimental results, the system averts the condensation in any case, and it spends a little more energy at maximum error point. In this situation, the temperature inside the car compartment is $30{ }^{\circ} \mathrm{C}$ and humidity is $30 \%$; however, such a real-time situation is seldom. Thus, we see that the maximum error point situation is not relevant.

\section{CONCLUSIONS}

This research presents one of the newly developed embedded systems, the ESCC, which provides good visibility for the car driver by preventing condensation on the car glasses, thereby improving the safety level.

In this study, a new device that uses the ESCC was proposed to control condensation on the glass. The software of this system was written in C language on an ATmega128L CPU, which is ideal for such designs. One of the major advantages of using the ATmega128L is that it has an 8-channel, 10-bit ADC on-chip, and it is inexpensive. The ADC was used as pin connections to the temperature and humidity sensors. Moreover, an LCD screen was installed to guarantee comfortable use of the ESCC.

Experimental results were in agreement with the simulation results. The plane equation was transformed using the dew-point table, and it was calculated in all possible situations. The real-time situation results were comparable with the simulation results in more than $50 \%$ of situations. The system was checked in critical situations and showed excellent quality. On the basis of experimental results, it can be seen that the designed ESCC was successful in achieving its design goal. The designed system was observed to function steadily from the beginning to the end. The algorithm of work satisfies the requirements by $96 \%$.

The ESCC performs well even in the case of maximum error of the transform plane equation. One of the main problems in this situation is that the ESCC spends more energy. However, it prevents condensation on the car glass $5{ }^{\circ} \mathrm{C}$ before the dew-point temperature is reached, whereas in an ideal system, the ESCC begins to heat the car glass 3 ${ }^{\circ} \mathrm{C}$ before reaching the dew-point temperature. The ESCC worked for $96 \mathrm{~h}$ without interruption, and all functions were performed successfully.

\section{ACKNOWLEDGMENT}

This research was supported by Yeungnam University research grants.

\section{REFERENCES}

[1] Ivica Crnkovic,"Component-based approach for embedded systems", 9th International Workshop on Component- Oriented Programming, Oslo, pp. 1-3, June 2004.

[2] Turley J., The two percent solution, embedded systems programming, http://www.embedded.com/story/ OEG20021217S0039, December 2002.

[3] Harvey M. Deitel and Paul J. Deitel, How to program $C, 2001$

[4] M. Morris Mano and Charles R. Kime, Logic and computer designfFundamentals- Third edition, pp. 127321, 2004

[5] Atmel.Atmel avr 8bit risc family. http://www.atmel. com/products/avr/.

[6] Atmega128 Datasheet, Web: http://www.atmel.com/dyn/ resources/prod_documents/doc2467.pdf

[7] http://en.Wikipedia.org/wiki/Dew_point 




Dmitriy Lee received the B.Sc. and M.Sc. degrees in Tashkent University of Information and Technology, Uzbekistan and Electronics Eng. from Yeungnam University, Korea, in 2004 and 2008, respectively. He was the member of the Institute of Information and Communication in Yeungnam University.

His current research interests are in sensor system.

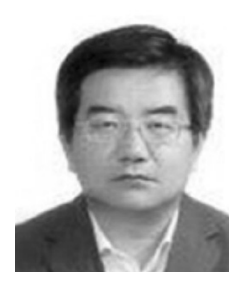

Neung-Ho Lee received his B.Sc. degrees in Electronics Eng. from Yeungnam University, Korea, in 1986. $\mathrm{He}$ is currently studying toward a Master's course in Electronics Eng., Yeungnam University. He also has worked as associate professor in the Department of Electronics and Telecommunications at Korea Polytechnics.

His main research interests are the design of biotelemeter, methods of communication and commercialization.

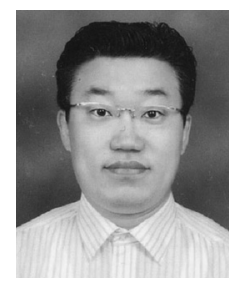

Yong-Wook Bae received the B.Sc. and M.Sc. degrees in Electronic Eng. from Yeungnam University, Korea, in 1996 and 1998, respectively. $\mathrm{He}$ is currently studying toward a doctoral course in Electronics Eng., Yeungnam University. From 2000 to 2007, he was an CIO with e-Hospital Korea Co., Daegu, where he worked on tele-EKG system and advised 30's general hospital to implement the hospital information system(HIS). He is currently a system design engineer with Jiyong Technlogy, Gumi, Korea.

His professional interests are in analog and mixed-signal circuit design and sensor system.

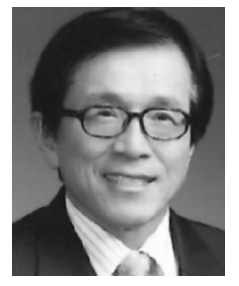

Hee-Don Seo received B.Sc. and Ph.D. degrees in Electronic Eng. from Yeungnam Univ., Korea, and Tohoku Univ., Japan, in 1973 and 1987, respectively.

Since 1987 he has been with the Dept. of Electronic Eng. of Yeungman Univ., where he is now a professor. He worked at the Samsung SDI from 1973 to 1980 . He was an associate professor in the Dept. Electrical and Electronic Eng. of Toyohashi Univ. of Technology, Japan for two years from 1992, and a visiting professor in the School of Biomedical Eng. of Virginia Univ. America for one year from 2001.

His current research interests are in Microsystem and Bio-electronics. 